\title{
Destruction of bulk ordering by surface randomness
}

\author{
D.E. Feldman ${ }^{1,2}$ and V.M. Vinokur ${ }^{1}$ \\ ${ }^{1}$ Materials Science Division, Argonne National Laboratory, 9700 South Cass, Argonne, Illinois 60439 \\ ${ }^{2}$ Landau Institute for Theoretical Physics, Chernogolovka, Moscow region 142432 Russia
}

\begin{abstract}
We demonstrate that the arbitrarily weak quenched disorder on the surface of a system of continuous symmetry destroys long range order in the bulk, and, instead, quasi-long range order emerges. Correlation functions are calculated exactly for the two- and three-dimensional $X Y$ model with surface randomness via the functional renormalization group. Even at strong quenched disorder the three-dimensional $X Y$ model possesses topological order. We also determine roughness of a domain wall in the presence of surface disorder.
\end{abstract}

75.10.Nr, 74.60.Ge, 05.50.+q, 64.60.Ak

The arbitrarily weak quenched disorder in the bulk of a system of continuous symmetry destroys long range order inherent to the pure system [1] provided that disorder breaks not only the translational symmetry but also the symmetry with respect to transformations of the order parameter, as e.g. random anisotropy in amorphous magnets does. This fundamental fact governs all the physics of condensed matter and results in a wealth of observed static and dynamics behaviors of real solids.

In many cases noticeable disorder presents only at the surface. Not surprisingly, surface randomness modifies the critical behavior near the surface [2], yet the common expectation is for the bulk properties to remain intact. In this Letter we show that arbitrarily weak surface disorder destroys long range order in the bulk of a system of continuous symmetry at the arbitrarily low temperature.

The predicted effect occurs in a rich variety of systems. Examples include crystal ordering in solids grown on a disordered substrate, liquid crystals interacting with an inhomogeneous surface, superconducting vortices pinned by surface impurities, etc. There are also many two-dimensional systems with edge randomness, e.g. superconducting films with columnar defects in a part of the film or films with a rough edge [3].

The reason as to why surface impurities, however weak, break long range bulk order is that the bulk contributes little to the energy of long-wave Goldstone modes: the surface random energy of long-wave excitations turns out to be greater than the corresponding bulk energy. As a result, the inhomogeneous state becomes favorable energetically. Note that ordering survives in the regions of the size less than the distance of these regions from the surface. In other words, if the distance between the two points is greater than their separation from the surface, the order parameter is different in those points. While long range order breaks down, topological order survives and quasi-long range order emerges. This means that the correlation length is infinite and that the correlation functions obey a slow logarithmic dependence of the distance.

An easy way to understand the main result of the Letter is based on Imry-Ma arguments [1, 何. Let us consider a region of size $L$ near the surface and compare the energies of ordered and disordered states of the region. If long range order is broken on the scales of the order of $L$ the loss in the bulk (elastic) energy is $E_{\mathrm{bulk}} \sim L^{D} / L^{2}$, where $D$ is the space dimension. The energy gain from the interaction with surface impurities scales as $E_{\text {surface }} \sim L^{(D-1) / 2}$. If $D<3$ then $E_{\text {surface }}>E_{\text {bulk }}$. Hence, the disordered state is favorable at $D<3$. On the other hand, long range order is favorable at $D>3$. The case $D=3$ is marginal and requires more quantitative consideration. We will see that in this case long range order is absent for the arbitrarily weak disorder similar to $D<3$.

We begin with the analysis of the exactly solvable Larkin model [1]. It is defined by the Hamiltonian

$$
H=\frac{J}{2} \int(\nabla \phi)^{2} d^{2} x d z-\int h(x) \phi(x, z=0) d^{2} x
$$

where $\phi$ is the order parameter, $h$ the random field, the $z$-axis is perpendicular to the disordered surface. At zero temperature we search for the energy minimum. To find the field configuration $\phi^{*}(x, z)$ at zero temperature we calculate the variation of the Hamiltonian (1) and make the Fourier transform with respect to the $x$ coordinates:

$$
J\left(q_{\|}^{2}-\partial_{z}^{2}\right) \phi^{*}\left(q_{\| \mid}, z\right)=h\left(q_{||}\right) \delta(z)
$$

Substituting the solution of this equation

$$
\phi^{*}\left(q_{\|}, z\right)=\phi^{*}\left(q_{||}, 0\right) \exp \left(-\left|q_{\|}\right| z\right)
$$

into Eq. (1) we get the energy 


$$
H^{*}=\int \frac{d^{2} q_{\|}}{(2 \pi)^{2}}\left[\left|q_{\|}\right|\left|\phi^{*}\left(q_{\|}, 0\right)\right|^{2}-h\left(q_{||}\right) \phi^{*}\left(-q_{\|}, 0\right)\right] .
$$

From Eq. (4) we find $\phi^{*}=\exp \left(-\left|q_{||}\right| z\right) h_{q_{||}} /\left(J\left|q_{||}\right|\right)$. Assuming that the random field $h$ is Gaussian and $\langle h(q) h(p)\rangle=$ $\Delta \delta(p+q)$ one finds the correlation function $G\left(r_{1}, r_{2}\right)=\left\langle\left(\phi\left(r_{1}\right)-\phi\left(r_{2}\right)\right)^{2}\right\rangle$. For example, if $z_{1}=z_{2} \ll\left(r_{1}-r_{2}\right)$ then

$$
G=\Delta /\left(\pi J^{2}\right) \ln \left(\left|x_{1}-x_{2}\right| / z_{1}\right)
$$

If $x_{1}=x_{2}, z_{1} \ll z_{2}$ then $G=\Delta /\left(2 \pi J^{2}\right) \ln \left(z_{2} / z_{1}\right)$. Since the correlation function is unlimited, there is no long range order. We will see that the same behavior is present in more complicated systems too, e.g. in the 3D $X Y$ model with surface disorder.

As the first nontrivial example we consider a domain wall in a media with disordered surface [5]. The domain wall is rough in the presence of bulk disorder 57. We show that surface disorder also makes it rough. The shape of the domain wall is described by the displacement of the wall as a function of $D-1$ coordinates $y=\phi(\mathbf{x}, z)$ where $\mathbf{x}$ and $z$ are the coordinates in a plane perpendicular to the surface, $z$ being the direction perpendicular to the surface. The Hamiltonian differs from (11) only by the dependence of the random contribution on $\phi$ : in the case of the random-bond disorder [5] it is a Gaussian $\delta$-correlated random variable $V(\mathbf{x}, \phi),\left\langle V\left(x_{1}, \phi_{1}\right) V\left(x_{2}, \phi_{2}\right)\right\rangle \sim \delta\left(x_{1}-x_{2}\right) \delta\left(\phi_{1}-\phi_{2}\right)$. We will demonstrate that the $\langle\phi \phi\rangle$ correlation function exhibits a power-law distance dependence:

$$
\left\langle\left(\phi\left(r_{1}\right)-\phi\left(r_{2}\right)\right)^{2}\right\rangle \sim\left|r_{1}-r_{2}\right|^{2 \zeta}
$$

Introducing the field $\phi^{*}\left(q_{\|}, z\right)$ as above and repeating the derivation of Eq. (何) we get

$$
H^{*}=\int \frac{d^{D-2} q_{\|}}{(2 \pi)^{D-2}} \frac{\left|q_{\|} \| \phi^{*}\left(q_{\|}, 0\right)\right|^{2}}{2}+\int d^{D-2} x V\left(x, \phi^{*}(x, 0)\right) .
$$

After replica averaging over disorder we get the effective replica Hamiltonian

$$
H_{R}=\int \frac{d^{D-2} q_{\|}}{(2 \pi)^{D-2}} \sum_{a} \frac{\left|q_{\|}\right|\left|\phi_{a}^{*}\left(q_{\|}, 0\right)\right|^{2}}{2 T}-\int d^{D-2} x \sum_{a b} \frac{R\left(\phi_{a}^{*}(x)-\phi_{b}^{*}(x)\right)}{2 T^{2}},
$$

where $a$ and $b$ are replica indices, $R$ is the function describing disorder. The problem can be studied with the functional renormalization group (RG) following the line of Ref. [6]. At each RG step we integrate out the momenta from the interval $1 /(a s)<q_{\|}<1 / a$, where $a$ is the ultraviolet cutoff and $s>1$, and make the rescaling $q_{\|} \rightarrow q_{\|} / s, x \rightarrow s x$, $\phi^{*} \rightarrow s^{\zeta} \phi^{*}$. The first term of Eq. (8) depends on $q_{\| \mid}$nonanalytically and hence does not renormalize [7]. Thus, the temperature obeys the $\mathrm{RG}$ equation $d T / d \ln L=(3-D-2 \zeta) T$ and we find a zero-temperature fixed point. Power counting shows that the whole function $R(\phi)$ is a relevant operator near $D=4$ in this fixed point. The RG equation for $R$ can be derived exactly in the same way as in Ref. [6] and to the first order in $\epsilon=4-D$ has the same form as in Ref. [6]

$$
d R(\phi) / d \ln L=(\epsilon-4 \zeta) R(\phi)+\zeta \phi R^{\prime}(\phi)+R^{\prime \prime}(\phi)^{2} / 2-R^{\prime \prime}(\phi) R^{\prime \prime}(\phi=0)
$$

where the factor $S_{2} /(2 \pi)^{2}=1 /(2 \pi)$ is absorbed into $R$. The solution of this equation can be read out of Ref. [6] and gives us $\zeta=0.2083 \epsilon$ in the correlation function (6). Using the result (3) for the displacement field $\phi$ one gets the same behavior $\left\langle\left(\phi\left(x_{1}, z_{1}\right)-\phi\left(x_{2}, z_{2}\right)\right)^{2}\right\rangle \sim\left|x_{1}-x_{2}\right|^{2 \zeta}$ in the bulk as at the surface provided that $\left|x_{1}-x_{2}\right|>z_{1} \sim z_{2}$. Thus, the domain wall is rough in 3 dimensions in the presence of surface disorder.

Next we consider the $X Y$ model with surface randomness. It is described by the same Hamiltonian (8) as the domain wall problem. However, due to the periodicity of the $X Y$ model the function $R(\phi)$ has period $2 \pi$. One can apply this model for the description of vortices in a superconductor with disordered surface [8]. The field $\phi$ describes displacements of the vortices from the regular positions in the Abrikosov lattice. Random potential is associated with impurities which pin vortices at the surface.

Due to the periodicity of the Hamiltonian there is no renormalization of the order parameter, i.e. $\zeta=0$. The RG equations have exactly the same structure as in the domain wall problem in dimension $D+1$. Since the dimension 4 is the critical dimension in the domain wall problem, the random $X Y$ model has the critical dimension $D=3$. Thus, we expect the logarithmic situation in 3 dimensions: the theory is asimptotically free and $R(\phi)$ can be represented as $R(\phi, L)=R^{*}(\phi) / \ln L$. The exact $\mathrm{RG}$ equation for $R^{*}$ has the same structure as the one-loop RG equation (9) at 
$\epsilon=1$ and $\zeta=0$. The analytical solution of this equation is known [8.9] and gives the following exact result for the correlation function:

$$
\left\langle\left(\phi\left(x_{1}, z_{1}\right)-\phi\left(x_{2}, z_{2}\right)\right)^{2}\right\rangle=\frac{\pi^{2}}{9} \ln \ln \left|x_{1}-x_{2}\right|
$$

at $\left|x_{1}-x_{2}\right|>z_{1}, z_{2}$. The possibility to obtain an exact result makes the 3D $X Y$ model with surface disorder a good candidate for a quantitative test of the functional RG. Since the correlation function is unlimited at large $r_{1}-r_{2}$, long range order is absent. However, the correlation function depends on the distance very slow. Such a situation can be described as super-quasi-long range order [9].

Our treatment of the $X Y$ model in terms of the elastic Hamiltonian (\$) is justified in the absence of topological defects. Similar to Ref. [9] we do not expect that the defects are relevant at weak disorder. At strong disorder, dislocations also do not proliferate deep in the bulk. Indeed, let us consider a dislocation of size $L$. The distance $R$ of any of its points from the surface is less than the dislocation length $L$ since otherwise the dislocation would not interact with the surface and should be energetically unfavorable. We estimate the energy gain associated with the disorder as $E_{\text {disorder }} \sim \sqrt{\Delta} \sqrt{L R}$, where $\Delta$ is the average square of the random field, and the bulk energy loss as $E_{\text {bulk }} \sim J L \ln R$. One sees that the bulk loss is greater than the surface gain for large $L$ even at strong disorder $\sqrt{\Delta} \gg J$. Thus, proliferation of large dislocation loops is unfavorable and the system renders topological order.

In two dimensions the $X Y$ model has only quasi-long range order at low temperatures even in the absence of disorder. Edge disorder modifies correlation functions but does not destroy quasi-long range order as we show below.

A recent experimental realization of a related two-dimensional system with edge randomness is a superconducting film with columnar defects in a part of it [3]. Vortices pinned by defects create a random potential near the boundary between the pure and impure parts of the film. This potential affects the vortex lattice in the pure part. An anisotropic film where vortices are free to move only in one direction can be described by the $X Y$ model with edge disorder [8].

One cannot use the $3-\epsilon$ expansion in this problem since no zero temperature fixed point exists in 2 dimensions: the scaling dimension of the temperature $\Delta_{T}=D-2$ becomes zero in two dimensions. Physically this result is related to the fact that thermal fluctuations have qualitatively the same effect on the ordering in the system as impurities: both destroy long-range order. Hence, we have to develop an RG procedure directly in two dimensions at nonzero temperature.

Similar to the derivation of Eq. (8) we obtain the following one-dimensional replica Hamiltonian for the field $\phi^{*}$ at the edge (in the absence of vortices)

$$
H_{R}=\int \frac{d q_{\|}}{(2 \pi)} \sum_{a} \frac{J\left|q_{\|}\right|\left|\phi_{a}^{*}\left(q_{||}, 0\right)^{2}\right|}{2 T}-\int d x \sum_{a b} \sum_{k} \frac{\Delta_{k} \cos k\left(\phi_{a}^{*}-\phi_{b}^{*}\right)}{T^{2}}
$$

where $k=n k_{\min }, k_{\min }$ being the smallest value of $k$ allowed by the symmetry, $n$ an integer. Note that in this expression $\phi^{*}$ is not a zero-temperature field configuration in contrast to the previous problems. The field $\phi$ in the bulk of our 2D system includes two contributions: one is related to $\phi^{*}$ via Eq. (3) and the other is a free thermally fluctuating field.

We first neglect vortices and then check how they change the behavior of the system. At $T>T_{c}=\pi J / k_{\min }^{2}$ all the nonlinear terms in $H_{R}$ are irrelevant. At high temperatures the correlation function is the same as in the absence of disorder and has the form $\left\langle\left(\phi^{*}\left(x_{1}\right)-\phi^{*}\left(x_{2}\right)\right)^{2}\right\rangle=2 T /(\pi J) \ln \left|x_{1}-x_{2}\right|$. Below $T_{c}$ the cosine term with the minimal $k$ becomes relevant.

At $k_{\min }=1$ we get the following one-loop RG equation

$$
\frac{d \Delta_{1} / T^{2}}{d \ln L}=\left(1-\frac{T}{\pi J}\right) \frac{\Delta_{1}}{T^{2}}-\frac{4 \Delta_{1}^{2}}{\pi^{4} J^{4}} a,
$$

where $a$ is the ultra-violet spacial cutoff. The fixed point solution of Eq. (11) is $\Delta_{1}^{*}=(1-T /(\pi J)) \pi^{2} J^{2} /(4 a)$. This result is valid, if the dimensionless combination $\Delta_{1} a / J^{2}$ is small.

To obtain the correlation function we add an infinitesimal contribution $\lambda\left|\phi_{q=k}^{a=1}\right|^{2} / T$ to the replica Hamiltonian (10). The correlation function $\left\langle\left|\phi_{k}^{1}\right|^{2}\right\rangle=-T d Z / d \lambda$, where $Z$ is the replica partition function. To find the contribution to $Z$ proportional to $\lambda$, we calculate $\phi$-independent corrections to $H_{R}(10)$ generated at each RG step. No $\lambda$-dependence is present in the first order in $\Delta_{1}$. Hence, we have to go to the second order in $\Delta_{1}$. This gives us the following result:

$$
\left\langle\left(\phi^{*}(x, z=0)-\phi^{*}(y, z=0)\right)^{2}\right\rangle=\frac{2 T}{\pi J} \ln \frac{|x-y|}{a}+\frac{\pi^{2}}{8}\left(1-\frac{T}{\pi J}\right)^{2} \ln \frac{|x-y|}{a}
$$


where the first term represents the effect of thermal fluctuations and the second one represents the effect of disorder. At $z>0$ the correlation function has the same structure (12), if $|x-y|$ is large enough. At $k_{\min }>1$ the result can be obtained from Eq. (12) by the transformation $\phi^{*} \rightarrow k \phi^{*}, T \rightarrow k^{2} T$.

The correlation function (12) is obtained in the vortex-free model. In the absence of disorder, the BerezinskyKosterlitz-Thouless transition occurs at $T_{\mathrm{BKT}}=\pi J / 2$. This temperature is lower than $T_{c}=\pi J / k_{\min }^{2}$ in the randomfield case $k_{\min }=1$. Thus, vortices lead to a breakdown of our result in the presence of the random-field disorder. What is the effect of vortices at $k_{\min }>1$ ? To answer this question we compare the bulk elastic energy of a vortex in the system of size $L$ and the energy of its interaction with the surface. The elastic energy $E_{\mathrm{bulk}} \sim J \ln L$. An estimation of the disorder energy must take into account the renormalization of the disorder strength at large scales. The fact that we obtain a fixed point $\Delta^{*}$ for $\Delta$ means that the effective disorder strength at scale $l$ is of order $\Delta^{*} a / l$ (rescaling at the RG steps!). The average square of the disorder energy scales hence as $E_{\text {dis }}^{2} \sim \int^{L} d l \Delta^{*} / l \sim \Delta^{*} \ln L$. There are $\sim L^{2}$ possible positions of the vortex. Assuming a Gaussian distribution for $E_{\mathrm{dis}}$ we get the following probability of such a disorder realization that the creation of a vortex is favorable: $p \sim L^{2} \exp \left(-J^{2} \ln ^{2} L /\left(\Delta^{*} \ln L\right)\right)$. One sees that $p \ll 1$, if $J^{2} \gg \Delta^{*}$. This justifies our vortex-free approximation and the result (12) at the vicinity of the critical temperature $T_{c}$ as the effective disorder strength $\Delta^{*}$ is small in that region.

What happens if both surface and bulk disorder is present? This question can be easily answered in the framework of the Larkin model [1]. Let the average square of the bulk random field be $\Delta_{\text {bulk. }}$. The correlation function in the presence of bulk disorder only [1] is $\left\langle\left(\phi\left(x_{1}\right)-\phi\left(x_{2}\right)\right)^{2}\right\rangle \sim \Delta_{\text {bulk }}\left|x_{1}-x_{2}\right| / J^{2}$. This should be compared with the correlation function in the presence of surface disorder only (5). One can see that the surface random field is the main source of order parameter fluctuations at scales $r=\left|x_{1}-x_{2}\right|<R_{c} \sim \Delta / \Delta_{\text {bulk }} \ln \left(\Delta /\left(\Delta_{\text {bulk }} a\right)\right)$. At scales $r>R_{c}$ bulk disorder dominates.

In conclusion, we demonstrate that even arbitrarily weak surface disorder destroys long range order in systems of continuous symmetry at any temperature. Topological order is not destroyed and quasi-long or super-quasi-long range order emerges. There are two quasi-long range ordered phases in the random two-dimensional $X Y$ model. The results of the Letter can be relevant for the characterization of disordered surfaces because the correlation functions of the system in contact with the surface contain information about the surface disorder.

We thank P.H. Kes and V.L. Pokrovsky for a useful discussion. This work was supported by the US DOE Office of Science under contract No. W31-109-ENG-38. DEF acknowledges support from RFBR grant No. 00-02-17763.

[1] A.I. Larkin, Sov. Phys. JETP 31, 784 (1970).

[2] A. Hanke and M. Kardar, Phys. Rev. Lett. 86, 4596 (2001).

[3] H. Pastorzia and P.H. Kes, Phys. Rev. Lett. 75, 3525 (1995); R. Besseling, T. Drose, V.M. Vinokur, and P. H. Kes (unpublished); N. Kokubo, R. Besseling, V.M. Vinokur, and P.H. Kes (unpublished); R. Besseling, Ph. D. Thesis, Leiden University (2001); M.V. Marchevsky, Ph. D. Thesis, Leiden University (1997).

[4] Y. Imry and S.K. Ma, Phys. Rev. Lett. 35, 1399 (1975).

[5] M. Mezard and G. Parisi, J. de Physique I 1, 809 (1991).

[6] D.S. Fisher, Phys. Rev. Lett. 56, 1964 (1986); L Balents and D.S. Fisher, Phys. Rev. B 48, 5949 (1993).

[7] J. Zinn-Justin, Quantum field theory and critical phenomena (Oxford University Press, Oxford, 1993).

[8] T. Nattermann and S. Scheidl, Adv. Phys. 49, 607 (2000); T. Giamrachi and P. Le Doussal, in Spin Glasses and Random Fields, edited by A.P. Young (World Scientific, Singapore, 1998) p. 321; e-print cond-mat/9705096.

[9] D.E. Feldman, Int. J. Mod. Phys. B 15, 2945 (2001). 